\title{
Constraints on radio source clustering towards galaxy clusters: application for cm-wavelength simulations of blind sky surveys.
}

\author{
Bartosz Lew \\ Toruń Centre for Astronomy, Nicolaus Copernicus University, \\ ul. Gagarina 11, 87-100 Toruń, Poland \\ email: blew@astro.uni.torun.pl
}

\begin{abstract}
We derive constraints on radio source clustering towards Planck-selected galaxy clusters using the NVSS point source catalog. The constraint can be used for making a more realistic Sunyaev-Zeldovich effect (SZE) mocks, calculating predictions of detectable clusters count and for quantifying source confusion in radio surveys.
\end{abstract}

Keywords. SZE, cosmological simulations, galaxy clusters, radio surveys

\section{Introduction}

Galaxy clusters are becoming observationally useful probes of cosmological models. In future thousands galaxy clusters will be detected via the SZE. Radio sources tend to correlate with galaxy cluster directions and constitute an important SZE contamination. The degree of the correlation and its redshift dependence remain uncertain due to poor statistics of faint radio source populations and insofar small SZE galaxy cluster samples. In Coble et al. (2007) a constraint on the source clustering was derived using the 28.5$\mathrm{GHz}$ survey yielding the radio source overabundance towards galaxy cluster centres a factor of 8.9 higher as compared to the clusters outskirts.

In preparation for the cm-wavelength sky surveys planned for RT32/OCRA-f (One Centimetre Receiver Array installed on 32-m radio telescope in Torun, Poland) and possibly for the envisaged Hevelius (a 100-m radio telescope) we calculated a possible scientific output from such blind surveys in terms of the number of detectable point sources and SZE detectable galaxy clusters using hydrodynamic simulations of large scale structure formation (Lew et al. (2014)). In that work we used the early Planck cluster sample (henceforth ESZ) (Planck Collaboration et al. (2011)) and we have shown that the point source clustering properties may significantly alter SZE counts predictions depending on the telescope beamwidth and observing frequency. In this report we revisit the point source clustering properties towards galaxy clusters using the extended cluster sample (henceforth PSZ) described in Planck Collaboration et al. (2014).

\section{Point source clustering from NVSS and Planck}

In Lew et al. (2014) we introduced a simple statistic to quantify the clustering of radio sources towards galaxy clusters as a function of angular distance $\theta_{\max }$ from cluster centres:

$$
\rho_{N}\left(\theta_{\max }\right)=\frac{1}{\pi \theta_{\max }^{2} N_{0}} \int_{0}^{\theta_{\max }} \frac{\partial N(\theta)}{\partial \theta} \mathrm{d} \theta \approx \frac{1}{\pi \theta_{\max }^{2} N_{0}} \sum_{i} A_{1}\left(\theta_{i}\right)
$$



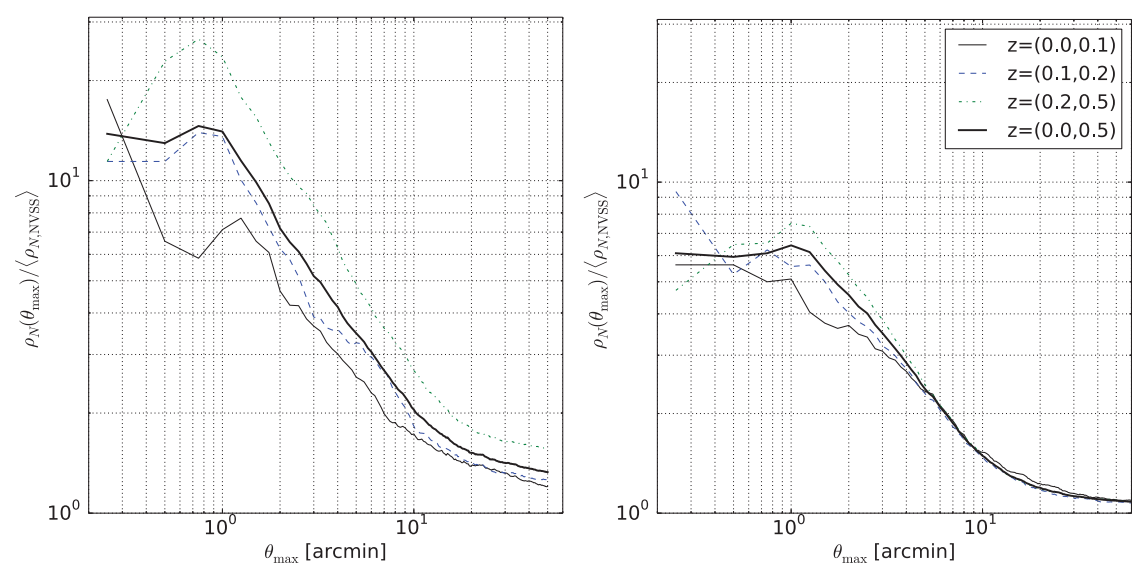

Figure 1. NVSS-normalised point source overdensity in ESZ (left) and PSZ (right) samples as a function of angular distance from the cluster centres. Individual lines represent overdensities for sub-samples selected according to the clusters redshift ranges.

where $\rho_{N}\left(\theta_{\max }\right)$ is the cumulative solid-angle source number density, $N_{0}$ is the total number of clusters in the sample and $A_{1}\left(\theta_{i}\right)=1$ if the radio source is within the angular distance $\theta_{\max }$ from its associated cluster's centre and $A_{1}=0$ otherwise. The summation extends over all radio sources.

We apply this statistic to the ESZ and PSZ samples which we cross-correlated with the $1.4 \mathrm{GHz}$ NVSS radio source catalogue (Condon et al. (1998)) out to $60^{\prime}$ form the cluster centres. In this cross-correlation study for ESZ (PSZ) sample 142 (993) out of 189 (1227) clusters were used. In Fig. 1 we plot the result relative to the NVSS average value of source density: $\left\langle\rho_{N, \mathrm{NVSS}}\right\rangle \approx 0.0135 \operatorname{arcmin}^{-2}$ and with $N_{0}=1$.

The point source overdensity relative to the cluster outskirts is clearly detected in the PSZ sample as well, but its peak value is systematically lower from the values inferred from ESZ sample in all redshift bins. It ranges between 5 and 10 within the innermost $1^{\prime}$. These lower values should be expected given that PSZ contains clusters yielding lower SZE flux densities, having lower masses and hence a lower source richness. A more detailed analysis and taking account of redshift space selections of radio sources (with redshifts determined via eg. optical identification) is needed to derive mass-richness scaling relations, while future wide-area $\mathrm{cm}$-wavelength surveys are required to understand a possible spectral dependence of the clustering. The clustering constraints are useful in making mock surveys more realistic especially when deriving survey confusion limits and the expected SZE cluster counts. $\dagger$

\section{References}

Coble, K., et al. 2007, AJ 897, 134 [astro-ph/0608274]

Condon, J. J., et al. 1998, AJ 1693-1716, 115

Lew, B., et al. 2014, submitted to JCAP [arxiv:1410:3660]

Planck Collaboration: Ade, P. A. R., et al. 2011, A\&SA A8, 536 [arxiv:1101.2024]

Planck Collaboration: Ade, P. A. R., et al. 2014, A\&SA accepted [arxiv:1303.5062]

$\dagger$ This work was financially supported by the Polish National Science Centre through grant DEC-2011/03/D/ST9/03373. A part of this project has made use of "Program Obliczeń WIElkich Wyzwań nauki i techniki" (POWIEW) computational resources (grant 87) at the Poznań Supercomputing and Networking Center (PSNC). 